\title{
Los factores determinantes de la eficiencia en la gestión de las Administraciones Tributarias Autonómicas españolas
}

\author{
Blanca AVEllón NARANJo a ${ }^{\text {a }}$ MARÍa José Prieto JANo ${ }^{\text {b }}$ \\ a Universidad de Valladolid, Facultad de Ciencias Sociales, Jurídicas y de la Comunicación, Plaza \\ de la Universidad, 1, 40005 Segovia, España.E-mail: blanca.avellon@uva.es \\ b Universidad de Valladolid, Facultad de Ciencias Económicas y Empresariales, Avda. Valle \\ Esgueva, 6, 47011 Valladolid, España. E-mail: jprieto@eco.uva.es
}

\begin{abstract}
RESUMEN
En esta investigación se estima la eficiencia técnica relativa de la gestión tributaria de las Administraciones Tributarias Autonómicas españolas de régimen común, en el periodo 2004 al 2012, mediante el Análisis Envolvente de Datos. Así mismo, se identifican los factores determinantes de la eficiencia mediante técnicas de Escalamiento Óptimo, como el Análisis de Correspondencias Simple. Los resultados más relevantes señalan que la eficiencia está correlacionada positivamente con la calidad de vida de los hogares y con el nivel educativo de la población. Además, cuando los partidos que gobiernan en la Comunidad Autónoma y en el Gobierno central difieren, la eficiencia gestora mejora.

Palabras clave: Administraciones Tributarias Autonómicas, gestión tributaria, factores de eficiencia, Análisis Envolvente de Datos, Análisis de Correspondencias Simple.
\end{abstract}

\section{The Determinants of Efficiency in the Tax Management of the Spanish Regional Tax Administrations}

\begin{abstract}
This research discusses the relative technical efficiency of tax management of Spanish regional tax Administrations of Common Regime, in the period 2004 to 2012, is estimated by Data Envelopment Analysis. Likewise, the determinants of efficiency are identified through Optimal Scaling techniques, such as Simple Correspondence Analysis. The most relevant results show that efficiency is positively correlated with the quality of life of households and with the educational level of the population. In addition, when the governing parties in the region and in the Central Government differ, the management efficiency improves.

Keywords: Spanish Regional Tax Administrations, Tax Management, Efficiency Factors, Data Envelopment Analysis, Simple Correspondence Analysis.
\end{abstract}

Clasificación JEL: H21, C10, C14

Artículo recibido en febrero de 2017 y aceptado en mayo de 2017

Artículo disponible en versión electrónica en la página www.revista-eea.net, ref. ə-35310 


\section{INTRODUCCIÓN}

En las últimas décadas, la evaluación de la actuación pública, es un tema que ha suscitado un gran interés en la comunidad académica y científica. Así, la medición de la eficiencia y la productividad en las instituciones públicas, se ha convertido en el objetivo prioritario de numerosos estudios, especialmente, en los momentos de crisis como los que hemos vivido en estos últimos años.

La técnica utilizada en la mayoría de estos trabajos sobre la medición de la eficiencia, ha sido el Análisis Envolvente de Datos -DEA-. La profusión de su utilización, fundamentalmente en Estados Unidos, Reino Unido y otros países desarrollados de nuestro entorno, ha supuesto que esta técnica se haya convertido en una herramienta esencial en el análisis de la optimización, en la prestación de los servicios públicos. Por mostrar algunas cifras, el número de artículos de investigación en los que se emplea la técnica, desde 1978 hasta la actualidad, ha ascendido a más de 4.000 trabajos; estos han sido presentados por alrededor de 2.500 autores; tanto en el ámbito nacional, como en el internacional. Las áreas más estudiadas han sido el sector bancario, la prestación del servicio de educación y la prestación del servicio de sanidad (Emrouznejad, Parker y Tavares, 2008).

El área de estudio de la presente investigación es la gestión del Sector Público; concretamente, la eficiencia de las Administraciones tributarias autonómicas españolas.

El estudio resulta de interés, en la medida en que las Administraciones son las encargadas de aplicar el sistema tributario; siendo este último, el responsable de generar la mayor parte de la recaudación, necesaria para financiar los servicios prestados por el Sector Público. Desde la Constitución de 1978 hasta la actualidad, España ha experimentado una fuerte descentralización de las competencias del Sector público. Esto supone, en términos generales, que la gestión de aproximadamente el 35 \% del gasto público, se realice por parte del gobierno intermedio; formado por los ejecutivos y los órganos de gestión de las Comunidades Autónomas -CCAA-. Estos gastos públicos incluyen, las políticas de sanidad, educación y los servicios públicos sociales; considerados de carácter esencial en la prestación del Estado del bienestar.

En este sentido, el análisis que se presenta es complejo, al referirse a la gestión administrativa tributaria autonómica, que está sujeta a diferentes procedimientos; a saber, la gestión, la recaudación y la inspección, básicamente. El correcto desarrollo de estos procedimientos, supone una gran responsabilidad por parte de los gestores encargados de su desempeño; que debe implicar la exigencia de la eficiencia en su aplicación. Precisamente, al tener encomendada la función primordial de recaudación de los tributos, los responsables tributarios no pueden permitir despilfarros en su gestión; debido a que esta recaudación representa la base de la financiación del Sector público autonómico. 
Dicho lo anterior, los objetivos que se plantean con esta investigación consisten en estimar los niveles de eficiencia técnica relativa, de las oficinas encargadas de la gestión tributaria de los tributos cedidos, de las diferentes CCAA de régimen común, en el periodo 2004-2012; para posteriormente, identificar las causas que han determinado esos niveles de eficiencia.

Este estudio supone una contribución al análisis de la eficiencia de la gestión tributaria descentralizada, que se ha abordado escasamente desde la doctrina hacendística; a la vez que ofrece una nueva evidencia empírica en el ámbito de la gestión tributaria autonómica. Así, con este estudio, se evalúa el proceso de cesión de competencias de gestión tributaria a las CCAA españolas.

De esta forma, la estimación de los niveles de eficiencia en la gestión de las Administraciones tributarias autonómicas, junto a un análisis de segunda etapa, que determine los factores que los ocasionan; puede ayudar al control de las Administraciones tributarias, aportando información relevante para los gestores tributarios, para lograr mejoras en sus resultados de gestión tributaria autonómica.

La identificación de los factores causantes de las diferencias, en la eficiencia de la gestión tributaria, puede ser de utilidad para los responsables tributarios, que deben rendir cuentas a los ciudadanos por sus niveles de eficiencia alcanzados.

Después de esta introducción, el resto del trabajo se estructura como sigue. En el segundo apartado se realiza una revisión de la literatura. En el tercer apartado se expone la metodología de la investigación, que consiste en la aplicación del DEA para estimar la eficiencia técnica; y la aplicación del análisis de correspondencias simple, para identificar los factores determinantes de la eficiencia en la gestión tributaria. En el cuarto apartado se realiza el análisis empírico aplicado a las Administraciones tributarias autonómicas españolas. En el quinto apartado se ofrecen los resultados. Finalmente, en el apartado sexto se establecen las conclusiones del análisis realizado, se ofrecen algunas recomendaciones de política económica, además se señalan las limitaciones que presenta la investigación.

\section{REVISIÓN DE LA LITERATURA: ANÁLISIS DE EFICIENCIA DE LA ADMINISTRACIÓN TRIBUTARIA}

Los estudios realizados sobre el análisis de la eficiencia y la productividad de la Administración tributaria, tanto en el contexto nacional como en el internacional, son escasos; a diferencia de lo que ocurre en otras áreas de la prestación de los servicios de la sanidad, la educación y los servicios de ámbito local, por parte del Sector público.

En las líneas que siguen se citan y se expone de manera sucinta, algunos de los trabajos que ofrecen una visión conjunta de la evaluación de la eficiencia y de la productividad de las Administraciones tributarias en el ámbito internacional y 
en los que, en términos generales se realiza un análisis de la eficiencia mediante métodos no paramétricos, o la aplicación de índices de productividad.

Estos estudios de eficiencia a nivel internacional, se han realizado en mayor medida en los países europeos; entre los que se encuentran los siguientes.

Moesen y Persoons (2002) estudian los factores influyentes en la eficiencia de las oficinas recaudatorias de Bélgica para el año 1991, mediante un DEA de dos etapas con una regresión Tobit y el Free Disponsal Hull -FDH- de Deprins, Simar y Tulkens (1984), obteniendo una relación entre la eficiencia y, la gestión a través una administración central y la cualificación del personal gestor. Barros (2005) analiza la eficiencia de las oficinas de recaudación de Lisboa en el periodo 19992002, mediante un análisis de frontera estocástica -SFA-, obteniendo una relación entre la eficiencia y la rigidez laboral, la ineficiencia $\mathrm{X}$ y las asimetrías en la información. Después, Barros (2006) modifica la investigación anterior y estima el índice de productividad de Malmquist -IPM- de Caves, Christensen y Diewert (1982). En una nueva modificación, Barros (2007), determina los factores influyentes en la eficiencia, obteniendo una relación positiva entre la eficiencia y el PIB, el gasto público y la ubicación de las oficinas urbanas. Katharaki y Tsakas (2010) estudian la eficiencia de las oficinas tributarias de Grecia para el periodo 2001-2006, mediante un DEA de dos etapas con una regresión Tobit. Determinan que la eficiencia está influenciada por el tamaño de escala y el PIB. También realizan un DEA-windows propuesto por Charnes et al. (1985) para generar estabilidad en el tiempo y detectar tendencias. Posteriormente, Katharaki y Tsakas (2014) modifican el estudio anterior, utilizando un DEA de dos etapas con una regresión Tobit, al que incorporan un método de simulación de datos para la inferencia estadística, el bootstrap propuesto por Simar y Wilson (1998). Forsund et al. (2006), analizan la eficiencia y el cambio productivo de las oficinas tributarias de Noruega para el periodo 2002-2004, mediante el DEA y el IPM, obteniendo que la productividad se relaciona con el tamaño de las oficinas. Posteriormente, Forsund et al. (2015), estiman el IPM con bootstrap para establecer intervalos de confianza, obteniendo que el cambio productivo en los tres años de análisis se distribuye entre una disminución del $26 \%$ y un aumento del $35 \%$, siendo el crecimiento promedio del $4 \%$.

Los estudios internacionales fuera de Europa, se han desarrollado en mayor medida en los países orientales; siendo escasos los aplicados a países latinoamericanos. Así, Jha et al. (1999) analizan los niveles de eficiencia en los estados de la India para los períodos 1980-1981 y 1992-1993, mediante un SFA, obteniendo una relación inversa entre la eficiencia y la riqueza. Thirtle et al. (2000) estudian la eficiencia en la recaudación en los estados de la India para el período 1980- 1992, mediante el IPM. Mattos et al. (2011) estudian la eficiencia de las oficinas de recaudación de Brasil para el 2004, aplicando un FDH con una segunda etapa para determinar los factores influyentes con una regresión 
Tobit, obteniendo una relación inversa entre la eficiencia y las prestaciones públicas. Ruy y Lee (2013) analizan la eficiencia de las oficinas tributarias de Corea para el periodo 1998-2011, mediante un DEA-windows.

Entre los estudios internacionales, destacan los estudios recientes que realizan un análisis comparativo entre las Administraciones tributarias de los países de la OCDE. Así, Alm y Duncan (2014), estudian la eficiencia de las oficinas de recaudación a nivel internacional para los países de la OCDE para el periodo 2007-2011, mediante un DEA de tres etapas con un SFA -utilizado por Fried et al. (2002) y Adam, Delis y Kammas (2011)-, para analizar el impacto sobre la eficiencia de los factores ambientales, que son la capacidad fiscal, las leyes fiscales y el grado de cumplimiento de los contribuyentes. Savić et al. (2015) analizan la eficiencia de las oficinas de recaudación a nivel internacional para algunos países de la OCDE para el periodo 2011-2012, mediante el DEA y un análisis de regresión; donde analizan la influencia de la eficiencia, el número de trabajadores de la administración tributaria y la tasa de empleo, sobre la economía sumergida.

En el ámbito nacional, por su parte, los trabajos son muy escasos en lo que se refiere a la medición de la eficiencia en la Administración tributaria española.

Entre estos estudios cabe destacar a González y Miles (2000) que analizan la eficiencia de las unidades regionales de inspección de la Agencia Estatal de Administración Tributaria -AEAT- de España para el 1995, mediante un DEA con bootstrap, obteniendo como resultado que la eficiencia media es del $81 \%$. Jiménez y Barrilao (2001), analizan la eficiencia de las delegaciones especiales de la Administración Tributaria española, para el año 1997, mediante el DEA, determinando las delegaciones referentes para el resto. Esteller (2003) analiza la eficiencia de las delegaciones tributarias territoriales, para los años 1992, 1995 y 1998, mediante un SFA; obteniendo como resultado que el déficit y la financiación incondicionada, afectan negativamente a la eficiencia. Fuentes (2008) analiza la eficiencia y la productividad para las oficinas tributarias de Alicante para el período 2004-2006, mediante un DEA Malmquist y quasiMalmquist -que tiene en cuenta el efecto dispar de la tecnología sobre los outputs-. Barrilao, Villar y Jiménez (2012) analizan la eficiencia gestora de las delegaciones especiales de la AEAT española para el año 2004, mediante el DEA, estableciendo un ranking de eficiencia. Posteriormente, Barrilao y Villar (2013) analizan la eficiencia de las oficinas tributarias especiales, para el año 2008, mediante un DEA. Fuentes y Lillo-Bañuls (2015), analizan el cambio productivo de las oficinas tributarias para el periodo 2004-2006, mediante el IPM con bootstrap y la prueba U de Mann Whitney, para estudiar la influencia de las variables del contexto; obteniendo que la población y el número de municipios influyen en la productividad. 


\section{METODOLOGÍA DE LA INVESTIGACIÓN}

\subsection{Estimación de la eficiencia técnica relativa: Análisis Envolvente de Datos}

El Análisis Envolvente de Datos -DEA- es una técnica de optimización matemática que permite la construcción de una superficie envolvente, frontera eficiente o función de producción empírica, a partir de los datos disponibles, en contextos de múltiples inputs y outputs, de un conjunto de DMU'S -decision making Units ó Unidades de toma de decisiones-. La técnica proporciona la estimación de la eficiencia relativa de cada una de las Unidades objeto de análisis.

El primer trabajo del DEA se publicó en 1978 por Charnes, Cooper y Rhodes (DEA-CCR). La medida de eficiencia obtenida coincide con la eficiencia radial de Farrel de 1957. Para Charnes et al. (1994), el DEA otorga a cada DMU una única medida de eficiencia relativa, optimizando la eficiencia de cada DMU en relación con las demás. Esta medida de eficiencia hace que sea posible identificar los factores de ineficiencia y la intensidad de los mismos. Además, el análisis pone de manifiesto las áreas de mejora de cada DMU, mediante la proyección de cada Unidad ineficiente a la frontera eficiente; permitiendo establecer un plan de actuación para la DMU al determinar sus objetivos -targets- de inputs y de outputs.

Se opta por la selección del DEA porque se ajusta a las actividades realizadas por el Sector público, donde no se dispone de información sobre los precios de los servicios prestados. El análisis permite analizar un conjunto de Unidades, que emplean simultáneamente múltiples inputs con los que obtienen múltiples outputs; sin imponer restricciones sobre la forma de la función de producción. Esta última razón, hace que se seleccione el DEA frente a los métodos frontera paramétricos, que requieren la especificación de una forma funcional concreta; cuyo primer modelo se debe a Feldestein (1967). Esta metodología paramétrica, puede ser tanto determinista -Aigner y Chu (1968) aplicaron el primer modelo frontera paramétrico determinista con una función de costes-; como estocástica, cuyo primer trabajo se debe a Aigner, Lovell y Schmidt (1977), también con el empleo de una función de costes.

La técnica del DEA presenta como principal limitación la sensibilidad a los datos utilizados, que pueden ser incorrectos o atípicos. Esta limitación se debe solventar con técnicas complementarias.

En esta investigación se emplea el modelo DEA - BCC (Banker, Charnes y Cooper, 1984) de rendimientos variables de escala y output orientado, en dos etapas, cuya formulación matricial se expone a continuación. 


$$
\begin{aligned}
\operatorname{máx}_{\varphi, \lambda, \mathrm{s}^{+}, \mathrm{s}^{-}} \mathrm{z}_{0}=\varphi+\varepsilon\left(\mathrm{Is}^{+}+\mathrm{Is}^{-}\right) \\
\text {s.a.: } \\
\lambda \mathrm{Y}=\varphi^{*} \mathrm{Y}_{0}+\mathrm{s}^{+} \\
\lambda \mathrm{X}=\mathrm{X}_{0}-\mathrm{s}^{-} \\
\overrightarrow{1} \lambda=1 \\
\lambda, \mathrm{s}^{+}, \mathrm{s}^{-} \geq 0
\end{aligned}
$$

En la expresión (1), $1 / \varphi^{*}=\theta^{*}$ es la puntuación de eficiencia técnica alcanzada por la entidad analizada, que representa la cantidad de producción máxima obtenida para el nivel de factor empleado. El vector de inputs y de outputs es $\mathrm{X}$ e $\mathrm{Y}$, respectivamente; siendo $\mathrm{X}_{0}$ e $\mathrm{Y}_{0}$, el vector de inputs y outputs de la entidad analizada. I, es la matriz identidad. El parámetro $\varepsilon$ es el infinitesimal más pequeño. El parámetro $\lambda$ identifica, para la entidad evaluada, las Unidades que actúan como benchmarking y que la envuelven. Los parámetros $\mathrm{s}^{+}$y s $^{-}$son las holguras ${ }^{1}$ asociadas a los inputs y a los outputs, respectivamente. La ecuación $\overrightarrow{1} \lambda=1$ es la restricción de convexidad.

La tecnología DEA ha evolucionado mediante la combinación con otras técnicas, paramétricas y no paramétricas, que añaden etapas al modelo original, cuyo cometido es el de ganar robustez en los indicadores de eficiencia estimados; al realizar un análisis de sensibilidad, incluir factores ambientales, así como las variables no controlables por el gestor. Para el cálculo de la sensibilidad y la estabilidad de los resultados de la eficiencia en el DEA, cabe destacar el modelo de Banker y Morey (1986), en el que incluyen factores controlables y no controlables en una sola etapa. Otra vía para ganar fiabilidad es el empleo de series temporales debido a la sensibilidad de la técnica DEA a las observaciones extremas (Trillo, 2002). También son de destacar los modelos multietápicos, a saber, el modelo de tres etapas propuesto por Fried et. al (2002), para el tratamiento de los factores exógenos.

En esta investigación, para solventar el problema de la dimensionalidad de la muestra y reducir los efectos de los datos atípicos, se aplica el modelo DEA-BCC como un DEA-windows (Charnes et al.; 1985), con una amplitud del subpanel coincidente con el panel total, que compara cada Administración tributaria autonómica con el resto y consigo misma, en cada uno de los años de estudio.

El modelo DEA temporal en forma de ventanas, consiste en agrupar los datos de un panel de T periodos, en intervalos de distinta amplitud. Posteriormente, se

\footnotetext{
${ }^{1}$ En Iglesias y Seijas (2008) se utiliza un modelo DEA basado en holguras. Según los autores, se obtienen puntuaciones de eficiencia más reducidas. Esto incrementa la discriminación del modelo.
} 
resuelve el problema envolvente de cada panel. El propósito de este análisis, es solucionar los problemas de dimensionalidad, cuando el número de variables inputs y outputs es elevado, en relación con el tamaño de las Unidades analizadas. También, es una técnica que mejora la estabilidad de la muestra en el tiempo y soluciona la presencia de outliers. Además de solucionar las dificultades anteriores, este tipo de metodología, tiene una importante ventaja, que es el aumento del poder discriminante, que se logra al incrementar el tamaño de la muestra. Esta última característica dota al modelo de la utilidad práctica que se busca en los análisis de eficiencia productiva, a saber, la clasificación en función de los niveles obtenidos.

La limitación de la técnica DEA-windows es que la tecnología de producción debe permanecer constante. Además, al obtener varios índices de eficiencia de la misma Administración tributaria en un solo panel, obliga a que se considere como índice de eficiencia, el valor medio (Trillo, 2002). Una derivación del modelo de ventanas, es el índice de productividad de Malmquist de Caves, Christensen y Diewert (1982), cuando los subpaneles son de 2 años.

La aplicación de un DEA-windows empleada en esta investigación, puede tener las mismas debilidades, respecto a la consideración de la tecnología de producción, que otros estudios recientes. Cabe citar como ejemplo, el trabajo de Alm y Duncan (2014); que utilizan las series temporales de los inputs y outputs en un solo programa DEA transversal ${ }^{2}$.

Sin embargo, el análisis permite comentar en qué medida las Administraciones utilizan los insumos para generar la cantidad de outputs.

\subsection{Determinación de los factores influyentes en la eficiencia técnica: Análisis de Correspondencias Simple}

En la segunda etapa de esta investigación, se analiza la relación existente entre los niveles de eficiencia alcanzados por las Administraciones tributarias autonómicas y un listado de variables influyentes, mediante técnicas de escalamiento óptimo. Concretamente, se emplea el análisis de correspondencias simple $-\mathrm{ACS}^{3}$-.

\footnotetext{
2 Alm y Duncan (2014, p. 13) promedian las variables inputs y outputs de los años 2007 al 2011, para aplicar el modelo DEA - BCC de forma transversal. Además, los autores anteriores sostienen que determinados inputs de un año concreto, afectan a la variable output -en su caso, recaudación por ingresos- en varios años; siendo incorrecto utilizarlo como insumo en un solo periodo de tiempo.

A nuestro juicio, la construcción de la frontera eficiente utilizando variables modificadas, en el caso anterior promediadas, no permite identificar si los resultados alcanzados en el análisis se deben a cambios en eficiencia, ó a cambios en productividad.

${ }^{3}$ La aplicación del ACS se realiza con los siguientes comandos. Se utiliza la medida de distancia Chi-cuadrado, porque con el empleo de la distancia euclídea, los términos con mayor valor
} 
El escalamiento óptimo ${ }^{4}$ es un conjunto de técnicas de reducción de la dimensión, que pueden ser empleadas para determinar la relación existente entre las variables. Entre estas técnicas, se encuentran el escalamiento multidimensional -EM ó multidimensional scaling, MDS-, métrico y no métrico; el análisis de correspondencias múltiple -ACM- y el análisis de correspondencias simple -ACS-.

El EM surge a principios del siglo XX en el campo de la psicología para estudiar la relación entre los estímulos físicos y los subjetivos. El EM es una técnica multivariante, que persigue objetivos similares al análisis factorial y al análisis cluster. Estos objetivos, consisten respectivamente, en reducir la dimensión de la muestra cuando las variables están correlacionadas; y en clasificar la muestra en grupos homogéneos, según las características similares de las variables.

El ACS es un caso particular del análisis en componentes principales, que se aplica a una matriz particular, y que se utiliza para el estudio de dos variables cualitativas. El ACS emplea una tabla de contingencia de las dos variables categóricas, donde se analiza la frecuencia absoluta de asociaciones entre las categorías; es decir, se analizan los supuestos en los que se dan a la vez las dos características de las variables. Mediante el valor propio se obtiene información de la correlación entre las puntuaciones de la fila y de la columna.

La representación gráfica de los puntos de las tablas de perfiles de filas y columnas -que son las proporciones marginales de la tabla de contingencia-, informa de la relación entre las categorías, a través de la proximidad de su localización en el gráfico, que es denominado diagrama de dispersión biespacial o mapa de correspondencias.

En esta investigación se emplea el ACS porque al tratarse de una técnica descriptiva se considera muy fiable. Además, se ajusta al objetivo perseguido en la investigación, que consiste en detectar los posibles factores condicionantes de la eficiencia técnica.

En la primera etapa de esta investigación, se estimada la variable eficiencia técnica mediante el DEA. Después, la eficiencia técnica se tabula en categorías para su mejor interpretación -según el baremo de Cooper, Seiford y Tone $(2007)^{5}$-. Además, algunas de las variables consideradas en la segunda etapa de

absoluto contribuyen más a la distancia. Se emplea el método de estandarización típico, donde se eliminan las medias de las filas y las columnas. Se elige el método de normalización simétrico, para analizar las diferencias entre las categorías de las dos variables.

${ }^{4}$ Para una información más completa se pueden consultar Pérez (2012) y Guerrero y Ramírez (2002).

${ }^{5}$ La eficiencia técnica se tabula en cuatro categorías: 1 , que representa a la eficiencia plena del $100 \%$; 2, que representa a la eficiencia alta, esto es, superior ó igual al $90 \%$; 3, que representa 
la investigación son cualitativas. En este contexto, la técnica del ACS se adecúa a las pretensiones del análisis, a saber, observar las posibles relaciones entre variables categóricas.

Se añade que, el ACS es superior en información a las tablas de contingencia, debido a que informa, tanto de la relación entre las variables, como de la relación entre las categorías de las variables.

Como limitaciones de la técnica se establece su incapacidad para determinar la independencia entre las variables explicativas; pero al ser tratadas por comparación en pares, esto no se produce.

Adicionalmente, para corroborar la relación entre las variables de segunda etapa y la eficiencia, se realiza la prueba chi-cuadrado de independencia entre variables, que determina en cada caso, la existencia de asociación. También se aplica este contraste, en los casos en los que no ha sido posible aplicar el ACS, por disponer de variables de dos categorías; debido a que no se pueden representar las dos dimensiones del gráfico de puntos de columna y fila.

\section{ANÁLISIS EMPÍRICO: ESTIMACIÓN DE LA EFICIENCIA TÉCNICA DE LAS ADMINISTRACIONES TRIBUTARIAS AUTONÓMICAS ESPAÑOLAS Y DETERMINACIÓN DE LOS FACTORES INFLUYENTES EN LA MISMA}

\subsection{Selección de la muestra}

El análisis de la eficiencia y la determinación de los factores condicionantes de esta, se aplica en las quince CCAA españolas financiadas mediante el régimen común, regulado en Ley Orgánica 3/2009, de 18 de diciembre, de financiación de las CCAA -LOFCA 3/2009-. El análisis comparativo requiere una muestra homogénea. Por lo anterior, se excluyen del estudio País Vasco, Navarra, Ceuta y Melilla.

La muestra de estudio está formada por un panel de 135 Unidades, formado por las quince Administraciones tributarias autonómicas, en cada uno de los años del periodo analizado, 2004-2012.

En esta investigación, se realiza una estimación de la eficiencia en la gestión de los tributos cedidos desde el Gobierno central a las CCAA; y gestionados por las mismas. Por lo tanto, los tributos cedidos incluidos en el análisis, son aquellos que otorgan autonomía tributaria a las Administraciones tributarias autonómicas, capacitándolas para ejercer las facultades gestoras sobre los mismos. Estos tributos son el Impuesto sobre transmisiones patrimoniales onerosas y actos jurídicos documentados -ITPAJD-, el Impuesto sobre sucesiones y donaciones -

a la eficiencia mínima aceptable, esto es, entre el 50 \% y el $90 \%$; y 4, que representa a los niveles muy ineficientes, esto es, inferior al $50 \%$. 
ISD-, los Tributos sobre el juego, el Impuesto sobre el patrimonio -IP-, el Impuesto especial sobre determinados medios de transporte -IEDMT- y el Impuesto sobre la venta minorista de determinados hidrocarburos -IVMDH- que desde el 1 de enero de 2013 se integra en el Impuesto especial sobre hidrocarburos.

Sin embargo, en el periodo analizado, las Administraciones tributarias autonómicas españolas no han comenzado a gestionar el IEDMT ni el IVMDH, por lo que no existen datos sobre la gestión descentralizada de los mismos.

El periodo analizado engloba dos Sistemas de financiación diferentes, el iniciado en 2002 y el iniciado en 2010; sin embargo, las diferencias en la cesta de tributos cedidos y gestionados por las CCAA son escasas, referidas al alcance y condiciones específicas de la cesión de los tributos. Por lo que, se mantienen los porcentajes de cesión y las competencias de gestión.

\subsection{Variables utilizadas para la estimación de la eficiencia en el DEA}

Las variables inputs y outputs utilizadas se obtienen del informe del Ministerio de Hacienda y Administraciones Públicas, que realiza la Inspección General. En este informe se publican los datos del factor trabajo y los resultados de la eficacia en la gestión de los tributos cedidos a las CCAA de Régimen Común. Se dispone de un panel de datos de 147 variables desagregadas por tributos; entre las que se encuentran, el personal encargado de realizar la gestión de los tributos cedidos -distribuido por grupos administrativos, áreas de gestión y tipo-, la gestión de declaraciones, la gestión de recursos interpuestos por los contribuyentes, la gestión de expedientes sancionadores, las reclamaciones económico-administrativas, el número de actas de inspección, las tasaciones periciales contradictorias y la recaudación tributaria, entre otras.

Para esta investigación se utilizan las variables que representan los resultados y los recursos, en las funciones gestoras relacionas con los tributos cedidos, para el periodo 2004-2012. De este modo, la variable input utilizada es el personal que se encarga de realizar las funciones de gestión de los tributos cedidos en la Comunidad Autónoma, medida en unidades de efectivos existentes a fin de año. Esta variable se obtiene como la agregación de tres variables, el personal propio de la Comunidad Autónoma -funcionario del Estado, funcionario de la Comunidad, interino y contratado-, el personal de las oficinas liquidadoras y el personal de las empresas de servicios. Es una serie homogénea que puede ser comparable entre CCAA.

No ha sido posible introducir otras variables de recursos, tales como el factor capital o la tecnología, por la ausencia de la publicación de los datos referidos a las mismas.

Para la selección de las variables outputs, se realiza un depurado de la 
información. Este proceso consiste en escoger, en un primer paso, las variables que miden el nivel de resultado alcanzado en cada uno de los procedimientos de gestión, recaudación, inspección y revisión en vía económico - administrativa; y que informan de la gestión de cada tributo cedido, de forma que se preseleccionan 21 variables outputs ${ }^{6}$.

Posteriormente, para adecuar las variables a la dimensión de la muestra de estudio, se realiza un análisis de correlación de Pearson al $5 \%$ de significatividad, por el que se reduce el número de variables outputs de 21 a 5, al eliminar la información redundante. Así, los outputs que participan en la investigación son la recaudación aplicada líquida, medida en miles de euros; la gestión de liquidaciones complementarias del IP, medida en unidades; la gestión de las Tasas sobre el juego, medida en unidades de declaraciones de casinos; el número de comprobaciones de valores declarados en las oficinas gestoras, las oficinas liquidadoras del distrito hipotecario y la Unidad facultativa de inspección, medido en unidades; y las actas de inspección instruidas por la Comunidad Autónoma, medida en unidades ${ }^{7}$.

Finalmente, se realiza un análisis exploratorio de los datos, para detectar los posibles valores extremos -outliers-, mediante diagramas de caja y bigotes y diagramas de control. No existen valores extremos en estas variables. Además, se realiza un análisis de casos faltantes -missing-, por el que se detecta un $6 \%$ de valores perdidos, en la variable gestión de tasas sobre el juego ${ }^{8}$. Esta preparación de los datos, previa a la aplicación del DEA, resulta fundamental para dotar de robustez a los indicadores de eficiencia obtenidos.

\subsection{Variables utilizadas para la determinación de los factores influyentes en la eficiencia técnica en el ACS}

Las variables de segunda etapa, condicionantes de la eficiencia gestora, pueden ser económicas, entre las que se encuentran, el PIB, la renta bruta disponible ajustada -RBDA-, la deuda pública y la desigualdad de la renta; fiscales, que son entre otras, los tipos impositivos, los ingresos por impuestos, la obligación de presentar el IP, la recaudación tributaria, el número de reclamaciones tributarias y el grado de cumplimiento fiscal; políticas, tales como la participación democrática, la ideología, las leyes fiscales y la discrepancia de

\footnotetext{
${ }^{6}$ Las variables pre-seleccionadas se ofrecen en la Tabla 4 de los anexos de la investigación.

${ }^{7}$ Los estadísticos descriptivos de las variables utilizadas se ofrecen en la Tabla 5 de los anexos.

${ }^{8}$ Existen 2 valores missing en 2004 y 2005 para la Comunidad Autónoma de Cantabria, debido a que no asume la gestión de las tasas sobre el juego hasta el año 2006, por el Acuerdo del 7 de diciembre de 2006. Puesto que en 2004 y 2005 la gestión sobre los tributos del Juego en Cantabria continúa siendo ejercida por la Agencia Estatal de Administración Tributaria, la solución para corregir los missing consistirá en emplear ceros para los valores faltantes; de otra forma, se estaría sobrevalorando la gestión de la Comunidad Autónoma.
} 
gobiernos en los distintos niveles del sector público; presupuestarias, como la capacidad fiscal, la autonomía fiscal y el gasto en educación; demográficas, que son entre otras, las que explican el comportamiento del contribuyente, como la edad, las cargas familiares, el estado civil, el sexo, el tamaño de la población de residencia (Domínguez, López y Rodrigo, 2014), el nivel de delincuencia y el nivel educativo; geográficas, que son entre otras, la superficie de la región, la temperatura media, los $\mathrm{m}^{2}$ de áreas verdes, el número de horas anuales con luz del día; y organizativas, como es la gestión realizada mediante la figura de la Agencia tributaria autonómica -ATA-.

Para la selección de las variables utilizadas en la segunda etapa del trabajo, se tienen en cuenta, las que se emplean en los estudios precedentes, aplicados a las Administraciones tributarias. Entre estos, se encuentra Barros (2007), que establece una relación positiva entre el PIB, el gasto público y la eficiencia. En Katharaki y Tsakas (2010) se determina la relación positiva entre el PIB y el tamaño de escala. También destaca el trabajo de Alm y Duncan (2014), donde consideran que las Administraciones tributarias operan en un ambiente definido por la capacidad fiscal, las leyes fiscales y el grado de cumplimiento voluntario de los contribuyentes. Estas variables están fuera del control de los administradores tributarios.

En esta investigación, también, se consideran las variables ambientales que definen el contexto económico, político y social en el que operan los agentes de las Administraciones tributarias; para observar la posible relación con los niveles de eficiencia obtenidos. Las variables utilizadas en la investigación son las siguientes ${ }^{9}$.

- Eficiencia técnica relativa tabulada en cuatro categorías, de cada Comunidad Autónoma y año 2004-2012; obtenida mediante el DEA.

- Gestión realizada mediante la figura de una ATA; obtenida de las leyes de creación de las Agencias ${ }^{10}$.

- Población en unidades de personas de cada Comunidad Autónoma y año 2004-2012, que representa el tamaño de la Comunidad Autónoma; obtenida del INE.

- Crecimiento de la población en porcentaje, de cada Comunidad Autónoma y año 2004-2012, que representa el crecimiento de la Comunidad

\footnotetext{
${ }^{9}$ Todas las variables monetarias de este trabajo, se han deflactado por el IPC en base 2011 para ser comparables entre periodos de tiempo. Las variables cuantitativas absolutas, se han relativizado por la población, para ser comparables entre regiones. Las variables de la investigación que no son cualitativas, se tabulan en categorías para aplicar el ACS. Se establece un máximo de 4 categorías en cada variable para que la lectura del gráfico de puntos sea factible.

${ }^{10}$ La gestión realizada mediante la figura de la ATA, se construye como una variable dicotómica dummy- que toma el valor 1 cuando la gestión de los tributos cedidos se realiza por una entidad en forma de ATA; y el valor 0 en el caso contrario.
} 
Autónoma; obtenida del INE.

- Número de reclamaciones económico - administrativas por tributos cedidos interpuestas en unidades por cada 10.000 habitantes de cada Comunidad Autónoma y año 2004-2012, que representa la calidad de la gestión tributaria; obtenida de los Presupuestos Generales del Estado.

- Coincidencia o discrepancia del partido político que gobierna en la Comunidad Autónoma y en el Estado; obtenida del portal oficial del Congreso de los diputados ${ }^{11}$.

- Obligación o no de presentar el IP; obtenida de la normativa del IP- ${ }^{12}$.

- RDBApc de las familias, que representa el nivel de desarrollo y la calidad de vida de los hogares; obtenida del INE-Contabilidad regional de España, en base 2008, serie disponible para 2008-2011.

- Delitos cometidos en unidades por cada 10.000 habitantes, de cada Comunidad Autónoma y año 2004-2012, que representa el carácter delictivo de la población; obtenida del INE.

- Nivel educativo alcanzado por el conjunto de la población de entre 16 a 64 años, medida con el porcentaje de población con estudios superiores, de cada Comunidad Autónoma y año 2004-2012; obtenida del INEIndicadores de calidad de vida ${ }^{13}$.

\section{RESULTADOS DE LA INVESTIGACIÓN}

\subsection{Resultados de la aplicación del Análisis Envolvente de Datos}

Los resultados del DEA se muestran en la Tabla 1, donde se ofrecen los estadísticos descriptivos de la variable eficiencia técnica para el período 20042012. Se observa que la eficiencia técnica relativa media de las Administraciones tributarias autonómicas españolas es del 77,94 \%; la mediana es del $81 \%$, que indica la eficiencia obtenida por la Unidad situada en la posición 68; la desviación

\footnotetext{
${ }^{11}$ Se construye una variable dicotómica -dummy- que toma el valor 1 cuando existe coincidencia entre los partidos políticos gobernantes; y el valor 0 en el caso de discrepancia.

${ }^{12}$ Se construye una variable cualitativa que representa el año de la presentación del $I P$, en lugar de considerar el ejercicio de vigencia del gravamen. La variable construida tomará el valor 1 en los ejercicios 2004 al 2008 y en 2012; el valor será 0 en 2009, 2010 y 2011.

${ }^{13}$ La variable se clasifica en Nivel 0-2 (hasta $1^{\circ}$ de secundaria), Nivel 3-4 (hasta postsecundaria no superior) y Nivel 5-8 (hasta doctorado). Un objetivo de la Estrategia Educación y Formación Europa 2020 es que al menos el 40 \% de la población entre 30 y 34 años haya alcanzado la educación superior. Por lo que, en esta investigación se utiliza el porcentaje de la población que obtiene el Nivel 5-8 (educación superior). Se construye una variable cualitativa que toma el valor 1 cuando el porcentaje de población con educación superior no alcanza el 20 \%; el valor 2 cuando el porcentaje está entre el $20 \%$ y 25 \%; y el valor 3, cuando el porcentaje es superior al $25 \%$.
} 
típica, es del $20 \%$, que indica la variabilidad de los niveles de eficiencia; el valor máximo es del $100 \%$, que informa de la obtención de la eficiencia plena en la muestra; y el valor mínimo es del 35,60 \%, que informa del nivel alcanzado por la Administración más ineficiente.

Tabla 1

Descriptivos de la variable eficiencia técnica relativa

\begin{tabular}{|c|c|c|c|c|}
\hline & & & Estadístico & Error Típ. \\
\hline \multirow{13}{*}{$\begin{array}{c}\text { variable } \\
\text { eficiencia } \\
\text { DEA-BCC } \\
2004-2012\end{array}$} & \multicolumn{2}{|l|}{ media } & 77,947 & 1,757 \\
\hline & \multirow{2}{*}{ intervalo de confianza media al $95 \%$} & límite inferior & 74,472 & - \\
\hline & & límite superior & 81,422 & - \\
\hline & \multicolumn{2}{|l|}{ media recortada al 5\% } & 78,970 & - \\
\hline & \multicolumn{2}{|l|}{ mediana } & 81,950 & - \\
\hline & \multicolumn{2}{|l|}{ varianza } & 416,793 & - \\
\hline & \multicolumn{2}{|l|}{ desv. típ. } & 20,415 & - \\
\hline & \multicolumn{2}{|l|}{ mínimo } & 35,600 & - \\
\hline & \multicolumn{2}{|l|}{ máximo } & 100,000 & - \\
\hline & \multicolumn{2}{|l|}{ rango } & 64,400 & - \\
\hline & \multicolumn{2}{|l|}{ amplitud intercuartil } & 38,130 & - \\
\hline & \multicolumn{2}{|l|}{ asimetría } & $-0,437$ & 0,209 \\
\hline & \multicolumn{2}{|l|}{ curtosis } & $-1,068$ & 0,414 \\
\hline
\end{tabular}

Fuente: Elaboración propia con SPSS.

En la Figura 1, se representa el histograma digital de la variable eficiencia técnica. Se observa que 40 Unidades analizadas, de la muestra de 135, son plenamente eficientes, debido a que los niveles de eficiencia técnica relativa obtenidos son de 100 puntos. Esto es, el 29,62 \% de la muestra analizada, realiza comportamientos óptimos en la gestión de los tributos cedidos, debido a que con la cantidad de factor trabajo que es empleado en las funciones de gestión tributaria, los outputs derivados de la misma, alcanzan el máximo nivel posible.

Estas Administraciones plenamente eficientes son Andalucía 2004, Andalucía 2006, Andalucía 2007, Andalucía 2008, Andalucía 2009, Andalucía 2010, Andalucía 2011, Andalucía 2012, Baleares 2005, Baleares 2006, Baleares 2007, Baleares 2008, Baleares 2010, Baleares 2011, Canarias 2004, Canarias 2006, Canarias 2007, Canarias 2008, Canarias 2009, Canarias 2010, Canarias 2011, Canarias 2012, Castilla y León 2004, Castilla y León 2005, Cataluña 2006, Cataluña 2007, la Rioja 2004, la Rioja 2005, la Rioja 2006, la Rioja 2007, la Rioja 2008, la Rioja 2010, Madrid 2005, Madrid 2006, Madrid 2007, Madrid 2008, Madrid 2009, Valencia 2010, Valencia 2011 y Valencia2012. 
Figura 1

Histograma digital de la variable eficiencia técnica relativa

\begin{tabular}{|rl|}
\hline Frequency & Stem \& Leaf \\
& \\
6,00 & 3. 556789 \\
5,00 & 4. 00023 \\
5,00 & 4. 66999 \\
5,00 & $5 \cdot 01223$ \\
6,00 & $5 \cdot 788899$ \\
16,00 & $6 \cdot 0011111222333444$ \\
10,00 & $6 \cdot 5666677888$ \\
5,00 & $7 \cdot 11134$ \\
6,00 & $7 \cdot 556678$ \\
11,00 & 8. 01112223444 \\
9,00 & 8. 566779999 \\
5,00 & $9 \cdot 01123$ \\
6,00 & $9 \cdot 557789$ \\
40,00 & 10. 0000000000000000000000000000000000000000 \\
Stem width: $10,00$. Each leaf: 1 case(s)
\end{tabular}

Fuente: Elaboración propia con SPSS.

Existen 8 CCAA que en algún momento han logrado la eficiencia plena; mientras que 7 nunca lo han logrado -Aragón, Asturias, Cantabria, Castilla la Mancha, Extremadura, Galicia y Murcia-. Las razones de ineficiencia para Aragón, Galicia y Murcia, se encuentran en que utilizan más personal gestor que la media; ya que obtienen niveles de output, en algunos casos, superiores a la media. A saber, en Aragón los niveles de recaudación son superiores, en Galicia se realizan más comprobaciones de valores y se instruyen más actas de inspección; y en Murcia, el nivel del número de comprobaciones de valores declarados es de los mayores.

Para las CCAA de Asturias, Cantabria, Castilla la Mancha y Extremadura, los niveles de factor trabajo son similares a la media. Por lo que en estos casos, la ineficiencia estriba en un nivel de output por debajo de la media. Así, en Asturias, la ineficiencia se debe al reducido número de comprobaciones de valores. En Cantabria, la causa de ineficiencia son las escasas liquidaciones complementarias del IP. Mientras que en Castilla la Mancha, no se alcanza el nivel medio de actas instruidas. Finalmente en Extremadura, la razón de la ineficiencia es el bajo nivel de recaudación, que está por debajo de la media.

\subsection{Resultados de la aplicación del Análisis de Correspondencias Simple}

Los resultados del análisis de la relación existente, entre los niveles de eficiencia estimados mediante el DEA, y las variables de segunda etapa, son los siguientes.

Los niveles de eficiencia técnica gestora alcanzados por las autonomías son superiores, cuando existe discrepancia entre el partido político gobernante en la Comunidad y el partido del Gobierno central. Por el contrario, cuando la 
Comunidad Autónoma tiene la misma orientación política que el Gobierno central, la Administración tributaria autonómica, se muestra más ineficiente en la gestión de los tributos cedidos. En la Tabla 2 se ofrece la tabla de contingencia de las variables eficiencia y coincidencia de Gobiernos, donde se aprecia la correlación entre las variables porque las frecuencias relativas por columnas son distintas. Además, para corroborar la relación, se realiza la prueba chi-cuadrado, por la que se rechaza la hipótesis nula de independencia entre las variables.

Tabla 2

Tabla de contingencia: variables eficiencia y coincidencia Gobierno autonómico y estatal

\begin{tabular}{|c|c|c|c|c|c|}
\hline & & & \multicolumn{2}{|c|}{$\begin{array}{l}\text { Coincidencia Gobierno } \\
\text { Autonómico y Estatal }\end{array}$} & \multirow[t]{2}{*}{ Total } \\
\hline & & & No coincide & Coincide & \\
\hline \multirow{16}{*}{ eficiencia } & \multirow{4}{*}{$\begin{array}{c}\text { eficiencia } \\
\text { plena } \\
100 \%\end{array}$} & recuento & 26 & 14 & 40 \\
\hline & & $\%$ dentro eficiencia & $65,0 \%$ & $35,0 \%$ & $100 \%$ \\
\hline & & $\%$ dentro coincidencia gobierno & $31,7 \%$ & $26,4 \%$ & $29,6 \%$ \\
\hline & & $\%$ total & $19,3 \%$ & $10,4 \%$ & $29,6 \%$ \\
\hline & \multirow{4}{*}{$\begin{array}{c}\text { eficiencia } \\
\text { alta } \\
{[90-100) \%}\end{array}$} & recuento & 9 & 2 & 11 \\
\hline & & $\%$ dentro eficiencia & $81,8 \%$ & $18,2 \%$ & $100 \%$ \\
\hline & & $\%$ dentro coincidencia gobierno & $11,0 \%$ & $3,8 \%$ & $8,1 \%$ \\
\hline & & $\%$ total & $6,7 \%$ & $1,5 \%$ & $8,1 \%$ \\
\hline & \multirow{4}{*}{$\begin{array}{c}\text { eficiencia } \\
\text { mínima } \\
\text { aceptable } \\
{[50-90) \%}\end{array}$} & recuento & 46 & 22 & 68 \\
\hline & & $\%$ dentro eficiencia & $67,6 \%$ & $32,4 \%$ & $100 \%$ \\
\hline & & $\%$ dentro coincidencia gobierno & $56,1 \%$ & $41,5 \%$ & $50,4 \%$ \\
\hline & & $\%$ del total & $34,1 \%$ & $16,3 \%$ & $50,4 \%$ \\
\hline & \multirow{4}{*}{$\begin{array}{c}\text { muy } \\
\text { ineficiente } \\
{[0-50) \%}\end{array}$} & recuento & 1 & 15 & 16 \\
\hline & & $\%$ dentro eficiencia & $6,2 \%$ & $93,8 \%$ & $100 \%$ \\
\hline & & $\%$ dentro coincidencia gobierno & $1,2 \%$ & $28,3 \%$ & $11,9 \%$ \\
\hline & & $\%$ del total & $0,7 \%$ & $11,1 \%$ & $11,9 \%$ \\
\hline \multirow{4}{*}{\multicolumn{2}{|c|}{ Total }} & recuento & 82 & 53 & 135 \\
\hline & & $\%$ dentro eficiencia & $60,7 \%$ & $39,3 \%$ & $100 \%$ \\
\hline & & $\%$ dentro coincidencia gobierno & $100 \%$ & $100 \%$ & $100 \%$ \\
\hline & & $\%$ total & $60,7 \%$ & $39,3 \%$ & $100 \%$ \\
\hline
\end{tabular}

Fuente: Elaboración propia con SPSS.

En el periodo analizado, 2004-2012, el 26,66 \% de las CCAA realizan la gestión tributaria mediante una ATA; que son, la Agencia tributaria de Andalucía -ATRIAN-, la Agencia tributaria de las Islas Baleares -ATIB-, la Agencia cántabra de administración tributaria -ACAT- y la Agencia tributaria de Cataluña. En la Figura 2, se observa la fecha de creación e inicio de las ATA’S.

No se ha detectado correlación estadísticamente significativa entre el nivel de eficiencia técnica gestora, y la realización de las funciones gestoras tributarias a través de una ATA. En la Tabla 3, se observa la independencia de las variables porque las frecuencias relativas condicionales por columnas son muy similares. 
Figura 2

Creación e inicio de la actividad de las ATA’S

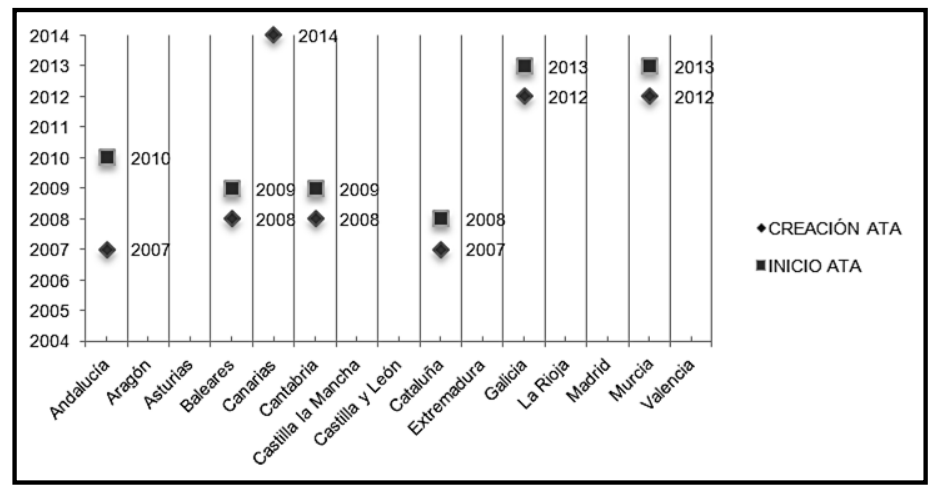

Fuente: Elaboración propia con Excel.

La falta de relación, hasta la fecha, entre la eficiencia y la gestión vía Agencia, puede ser debida a que se trata de un proceso relativamente reciente. Así mismo, el resultado puede estar ocasionado por los fenómenos de inercia de las formas de gestión anteriores a la gestión mediante las Agencias; unidos a la falta de experiencia gestora a través de este organismo. Del mismo modo, los efectos de la gestión a través de las Agencias, podrían estar sometidos a dilaciones en el tiempo, por lo que se podría considerar un estudio futuro, cuando este nuevo organismo gestor se haya consolidado, y se disponga de suficientes datos que recojan su actividad.

Tabla 3

Tabla de contingencia: variables eficiencia y actividad realizada por una Agencia

\begin{tabular}{|c|c|c|c|c|c|c|c|}
\hline & & & \multicolumn{4}{|c|}{ eficiencia } & \multirow[b]{2}{*}{ total } \\
\hline & & & $\begin{array}{c}\text { eficiencia } \\
\text { plena } \\
100 \%\end{array}$ & $\begin{array}{c}\text { eficiencia } \\
\text { alta } \\
{[90-100) \%}\end{array}$ & $\begin{array}{l}\text { eficiencia } \\
\text { mínima } \\
\text { aceptable } \\
{[50-90) \%}\end{array}$ & $\begin{array}{c}\text { muy } \\
\text { ineficiente } \\
{[0-50) \%}\end{array}$ & \\
\hline \multirow{8}{*}{ 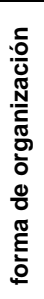 } & \multirow{4}{*}{ 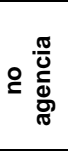 } & recuento & 35 & 9 & 60 & 15 & 119 \\
\hline & & $\%$ dentro no agencia & $29,4 \%$ & $7,6 \%$ & $50,4 \%$ & $12,6 \%$ & $100 \%$ \\
\hline & & $\%$ dentro eficiencia & $87,5 \%$ & $81,8 \%$ & $88,2 \%$ & $93,8 \%$ & $88,1 \%$ \\
\hline & & $\%$ total & $25,9 \%$ & $6,7 \%$ & $44,4 \%$ & $11,1 \%$ & $88,1 \%$ \\
\hline & \multirow{4}{*}{$\frac{\frac{\pi}{0}}{\frac{C}{0}}$} & recuento & 5 & 2 & 8 & 1 & 16 \\
\hline & & $\%$ dentro agencia & $31,2 \%$ & $12,5 \%$ & $50 \%$ & $6,2 \%$ & $100 \%$ \\
\hline & & $\%$ dentro eficiencia & $12,5 \%$ & $18,2 \%$ & $11,8 \%$ & $6,2 \%$ & $11,9 \%$ \\
\hline & & $\%$ total & $3,7 \%$ & $1,5 \%$ & $5,9 \%$ & $0,7 \%$ & $11,9 \%$ \\
\hline \multirow{4}{*}{\multicolumn{2}{|c|}{ total }} & recuento & 40 & 11 & 68 & 16 & 135 \\
\hline & & $\%$ dentro agencia & $29,6 \%$ & $8,1 \%$ & $50,4 \%$ & $11,9 \%$ & $100,0 \%$ \\
\hline & & $\%$ dentro eficiencia & $100 \%$ & $100 \%$ & $100 \%$ & $100 \%$ & $100 \%$ \\
\hline & & $\%$ total & $29,6 \%$ & $8,1 \%$ & $50,4 \%$ & $11,9 \%$ & $100 \%$ \\
\hline
\end{tabular}

Fuente: Elaboración propia con SPSS. 
También, se ha comprobado que existe una correlación positiva débil entre el nivel de eficiencia en la gestión tributaria y el número de delitos cometidos en las CCAA; según muestra el coeficiente de correlación de Pearson obtenido de 0,228 puntos; siendo la significación bilateral de 0,008 puntos. La estructura y las características de la población de cada Comunidad Autónoma, se pueden asociar, aunque de forma muy débil, con la forma de actuar por parte de las Administraciones tributarias autonómicas; que implica que cuanto peores son las condiciones a las que se enfrentan los funcionarios públicos para gestionar los impuestos, mayores son los niveles de eficiencia y el buen hacer en sus labores administrativas.

Los resultados del ACS se presentan en la Figura 3, que representa las proporciones marginales de las frecuencias de cada categoría. Se observa la proximidad de las categorías eficiencia plena y carácter delictivo alto. Las Administraciones en las que el número de delitos cometidos es medio, entre 40 y 60 delitos por cada 10.000 habitantes, presentan un nivel estimados de eficiencia mínima aceptable. Los niveles de gestión muy ineficientes se asocian con baja delincuencia. Sin embargo, la relación entre las categorías, alta eficiencia y bajo nivel de delincuencia, es contraria a esta tendencia, por el reducido grado de asociación entre las variables, según determina el coeficiente de contingencia obtenido de un $32 \%$.

Figura 3

Gráfico de puntos de columna y de fila: variables eficiencia y carácter delictivo de la población

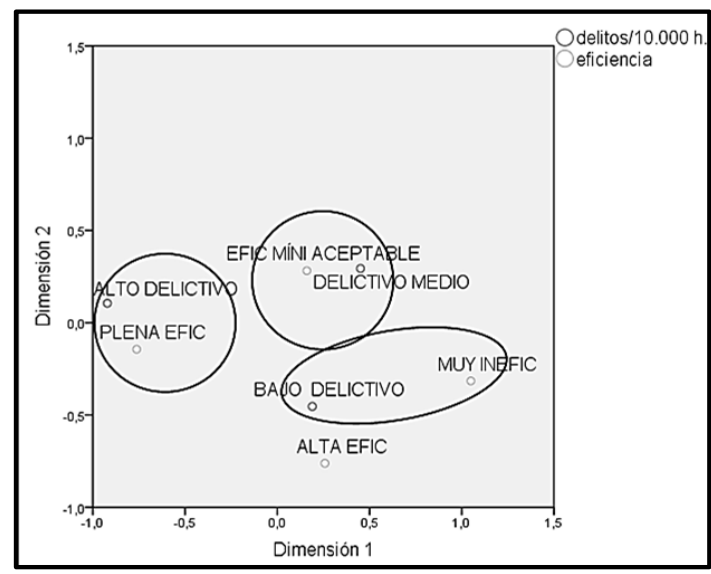

Fuente: Elaboración propia con SPSS.

Así mismo, se ha detectado la existencia de correlación positiva entre la eficiencia técnica y el nivel educativo de la sociedad española, que puede influir en el nivel de renta, la salud, el empleo, la integración social y el grado de 
cumplimiento fiscal de los contribuyentes, afectando positivamente al grado de éxito alcanzado por las Administraciones tributarias autonómicas.

Los resultados del ACS se presentan en la Figura 4. La asociación entre las categorías de las variables es la siguiente. La eficiencia plena se asocia con las CCAA con un nivel educativo medio -el porcentaje de población con educación superior está entre el $20 \%$ y el $25 \%$-. La eficiencia alta y los niveles de eficiencia mínima aceptable, se corresponden con regiones cuyo nivel educativo es alto, esto es, superior al $25 \%$. La gestión tributaria muy ineficiente se asocia con un nivel educativo bajo, es decir, la población con educación superior es inferior al $20 \%$.

Figura 4

Gráfico de puntos de columna y de fila: variables eficiencia y nivel educativo

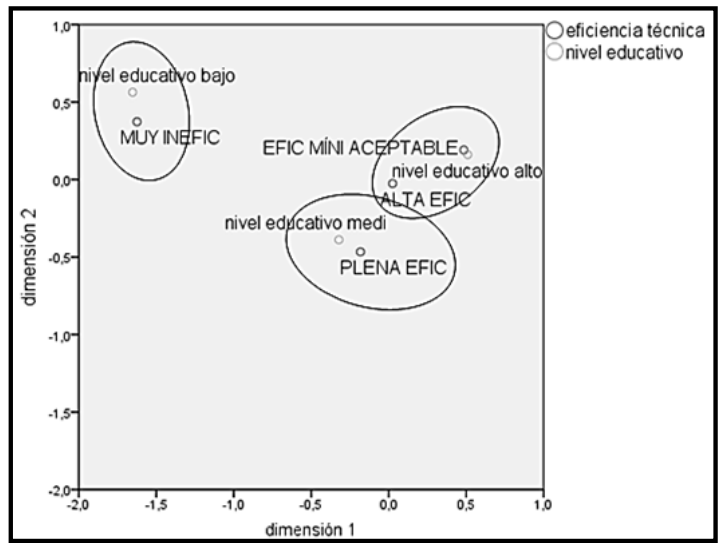

Fuente: Elaboración propia con SPSS.

Del mismo modo, la investigación determina que un mayor nivel de desarrollo económico y la calidad de vida de los hogares españoles, medido con la RBDApc, es un condicionante positivo para lograr un mayor nivel de eficiencia en la gestión tributaria por parte de las Administraciones tributarias Autonómicas.

Los resultados del ACS se presentan en la Figura 5. Se relacionan las categorías gestión muy ineficiente con RBDApc baja; inferior a 18.000 euros per cápita. Las categorías de eficiencia mínima aceptable y altamente eficiente se asocian con una RBDApc media; situada entre 18.000 y 20.000 euros per cápita. La eficiencia plena se asocia con la RBDApc alta; que excede de 20.000 euros per cápita.

No se ha detectado correlación entre los niveles de eficiencia productiva de las Administraciones tributarias autonómicas españolas y la obligación de presentar del IP; por tanto, con la realización de las actividades de gestión relacionadas con el mismo. La reducción de la carga de trabajo, en los ejercicios 2009 a 2011, y el 
posterior incremento de la misma, en 2012, informa de la capacidad de adaptación de los medios personales encargados de la gestión tributaria.

Figura 5

Gráfico de puntos de columna y de fila: variables eficiencia y RBDApc

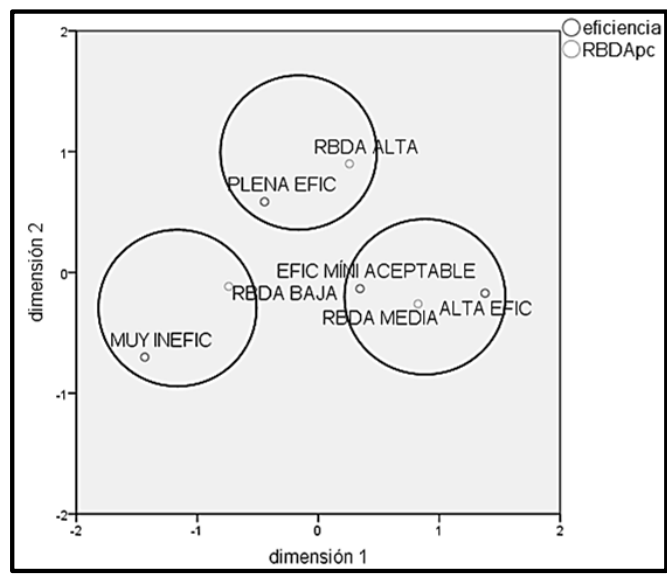

Fuente: Elaboración propia con SPSS.

Tampoco se determina la existencia de relación, entre la eficiencia y la población; que representa el tamaño de las CCAA. De la misma forma, no se encuentra asociación entre la eficiencia y el crecimiento de la población de la Comunidad Autónoma. Esto puede deberse, a que se aplica un modelo DEABCC, de rendimientos variables a escala, que estima la eficiencia técnica pura, que ya tiene corregidos los efectos de escala.

Finalmente, la investigación determina que no existe correlación entre la eficiencia técnica y las reclamaciones económico-administrativas por los tributos cedidos interpuestas por los contribuyentes; que puede ser un indicativo de la calidad gestora. La causa fundamental de la imposición de reclamaciones es la falta de motivación en la valoración de los bienes objeto de gravamen. Según esta investigación, este desacuerdo entre la Administración y el contribuyente, no influye en los niveles de eficiencia técnica alcanzados por las Administraciones.

\section{CONCLUSIONES, RECOMENDACIONES DE POLÍTICA ECONÓMICA Y LIMITACIONES DE LA INVESTIGACIÓN}

Según el DEA-BCC output orientado, aplicado en esta investigación, la eficiencia técnica relativa de las Administraciones tributarias autonómicas españolas, en el desempeño de la gestión de los tributos cedidos, para el periodo 2004 - 2012, es del 77,94 \% por término medio. Este resultado supone que para el logro de la eficiencia técnica plena, deberían expandir los outputs de forma 
radial en una media del 28,30 \%. Entre estos productos, se encuentra el aumento de la recaudación, del número de valoraciones realizadas por los funcionarios tributarios, y del número de liquidaciones gestionadas del IP, entre otros.

Los factores que se han mostrado determinantes de la eficiencia técnica administrativa -según el ACS y en su defecto la prueba de independencia de la chi- cuadrado-, son el nivel de desarrollo y la calidad de vida de los hogares medido con la RBDApc-, el carácter delictivo de la población -medido con el número de delitos-, y el nivel educativo de la población -medido con el porcentaje de población con educación superior-.

Asimismo, se obtiene un mayor nivel de eficiencia técnica, cuando el partido gobernante en la Comunidad Autónoma difiere del partido político del Gobierno estatal.

Estos resultados, ponen de manifiesto que el contexto económico, sociopolítico y socio-demográfico es determinante en los niveles de gestión alcanzados por los funcionarios de las Administraciones tributarias autonómicas españolas.

Por lo anterior, para mejorar los niveles de eficiencia en la gestión de los tributos cedidos, los responsables tributarios podrían desarrollar políticas públicas expansivas; focalizadas al incremento del nivel educativo, al crecimiento económico de la Comunidad Autónoma y al fomento del desarrollo económico de la ciudadanía.

Por otra parte, los autores son conscientes de las limitaciones de la investigación, que se comentan a continuación.

El DEA es una técnica sensible a los datos. En un intento por suavizar la muestra de datos, se utiliza el modelo DEA-windows y se realiza un análisis previo de outliers. Con esta variante de la metodología DEA, se obtienen unas ventajas, ya que se elimina el problema de la dimensionalidad -presente en esta investigación-, se aumenta el carácter discriminatorio del modelo y se soluciona la presencia de errores de medida en los datos o la existencia de outliers, haciendo los indicadores de eficiencia más robustos.

Sin embargo, la principal limitación de esta metodología DEA-windows, es que supone la ausencia de cambio productivo. La técnica no es capaz de identificar las razones de los indicadores de eficiencia obtenidos - productivas o de eficiencia -; aunque es determinante para utilizar los niveles de eficiencia en términos comparativos. Por esto, las estimaciones son susceptibles de utilizarse para establecer un ranking de gestión tributaria.

Por lo anterior, se realiza una segunda etapa, con un ACS aplicado a los estimadores de eficiencia, para identificar esos factores influyentes.

Una derivación del DEA-windows es el IPM para una amplitud del panel de datos, de 2 años. Por motivos de extensión, este análisis será abordado en otra investigación. 


\section{REFERENCIAS BIBLIOGRÁFICAS}

ADAM, A., DELIS, M. y KAMMAS P. (2011). "Public Sector Efficiency: Leveling the Playing Field between OECD Countries". Public Choice, 146(1), pp. 163-183.

AIGNER, D.J. y CHU, S. (1968). "On estimating the industry production function". American Economic Review, 58, pp. 826-839.

AIGNER, D.J.; LOVELL, C.A. y SCHMIDT, P. (1977). "Formulation and estimation of stochastic frontier production function models". Journal of Econometrics, 6, pp. 21-37.

ALM, J. y DUNCAN, D. (2014). "Estimating Tax Agency Efficiency". Public Budgeting and Finance, 34(3), pp. 92-110.

BANKER, R. D.; R. C. MOREY (1986). "Efficiency analysis for exogeneously fixed inputs and outputs". Operations Research, 34(4), pp. 13-21.

BANKER, R.D.; CHARNES, A. y COOPER, W.W. (1984). "Some Models for Estimating Technical and Scale Inefficiencies in DEA". Management Science, vol. 30(9), pp. 1078-1092.

BARRILAO, P.; VILLAR, E. (2013). "The efficiency of the regional management centers of the tax administration in Spain". Journal of US-China Public Administration, 10(1), pp. 49-56.

BARRILAO, P.; VILLAR, E. y JIMÉNEZ, J.D. (2012). "La eficiencia de la Administración Tributaria en España". XV Encuentro de Economía Aplicada, A Coruña, June 7-8 2012.

BARROS, C.P. (2005). "Performance measurement in tax offices with a stochastic frontier model". Journal Economics Studies, 32(6), pp. 497-510.

BARROS, C.P. (2006). "Measuring total productivity in Lisbon tax offices with a Malmquist index". Tijdschrift voor Economie Management, 51(1), pp. 25-46.

BARROS, C.P. (2007). "Technical and allocative efficiency of tax offices: a case study". International Journal of Public Sector Performance Management, 1(1), pp. 41-61.

CAVES, D.W.; CHRISTENSEN, L.R. y DIEWERT, W.E. (1982). "The economic theory of index numbers and the measurement of input, output and productivity". Econometrica, 50(6), pp. 1393-1414.

CHARNES, A.; COOPER, W.W. y RODHES, E. (1978), "Measuring the efficiency of Decision Making Units". European Journal of Operational Research, 2, pp. 429-444.

CHARNES, A.; et al. (1985). "Foundations of Data Envelopment Analysis for ParetoKoopmans efficient empirical production functions". Journal of Econometrics, 30, pp. 91-107.

CHARNES, A.; et al. (1994). Data Envelopment Analysis: Theory, Methodology and Applications. Estados Unidos: Springer Science and Business Media.

COOPER, W.; SEIFORD, L.M. y TONE, K. (2007). Data Envelopment Analysis: A comprehensive text with models, Applications, references and DEA-Solver Software, ( $2^{\mathrm{a}}$ ed.) Estados Unidos: Springer Science and Business Media.

DEPRINS, D.; SIMAR, L. y TULKENS, H. (1984). "Measuring Labor Inefficiency in Post Offices". En MARCHAND, M.; PESTIEAU; P. y TULKENS, H. (eds.): The Performance of Public Enterprises: Concepts and Measurements. Nueva York: North - Holland. 
DOMÍNGUEZ, F.; LÓPEZ, J. y RODRIGO, F. (2014). "El hueco que deja el diablo: Una estimación del fraude en el IRPF con microdatos tributarios". Informe de la Fundación de Estudios de Economía Aplicada (FEDEA), Estudios sobre la Economía Española, EEE2014-01.

EMROUZNEJAD, A.; PARKER, B. y TAVARES, G. (2008). "Evaluation of research in efficiency and productivity: A survey and analysis of the first 30 years of scholarly literature in DEA". Review Socio - Economic Planning Sciences, 42, pp. 151-157.

ESTELLER, A. (2003). "La eficiencia en la administración de los tributos cedidos: un análisis explicativo". Papeles de Economía Española, 95, pp. 320-334.

FARREL, M.J. (1957). "The Measurement of Productive Efficiency". Journal of the Royal Statistical Society, Series A, general, vol. 120(3), pp. 253-281.

FERNÁNDEZ, R. y GARCÍA, M. A. (2010) "Empresas públicas autonómicas: un gobierno a la sombra del gobierno". Institut d'Economía de Barcelona, Universitat de Barcelona, Document de treball 2010/6, pp. 126-135.

FELDESTEIN, M. (1967). "Specification of the Labor Input in the Aggregate Production Function". Review of Economic Studies, 1967. 34(4), pp. 375-386.

FORSUND, F.R.; et al. (2006). "The tax man cometh- but is the efficient?". National Institute Economic Review, 197, pp. 106-119.

FORSUND, F.R.; EDVARDSEN, D.F. y KITTELSEN, A.C. (2015). "Productivity of tax offices in Norway". Journal Productivity Analysis, 43, pp. 269-279.

FRIED, H.; et al. (2002). "Accounting for Environmental Effects and Statistical Noise in Data Envelopment Analysis". Journal of Productivity Analysis, 17(1) pp. 157-174.

FUENTES, R. (2000). Eficiencia de los centros públicos de educación secundaria de la provincia de Alicante. Tesis Doctoral, Departamento de Análisis Económico Aplicado, Facultad de Ciencias Económicas, Universidad de Alicante.

FUENTES, R. (2008). "Productivity at the SUMA tax offices". En VVAA: Proceedings of the $11^{\text {th }}$ Toulon-Verona International conference on quality in services, en Firenze University Press. pp. 323-333.

FUENTES, R. y LILLO-BAÑULS, A. (2015). "Smoothed bootstrap Malmquist index based on DEA model to compute productivity of tax offices". Expert systems with applications, 42, pp. 2442-2450.

GONZÁLEZ, X.M. y MILES, D. (2000). "Eficiencia en la inspección de hacienda”. Revista de Economía Aplicada, 24(vol. VIII), pp. 203-219.

GUERRERO, F. M. y RAMíREZ, J.M. (2002). "El análisis de escalamiento multidimensional: una alternativa y un complemento a otras técnicas multivariantes". X Jornadas Madrid 2002 de ASEPUMA (Asociación Española de Profesores Universitarios de Matemáticas aplicadas a la Economía y la Empresa)

HUNTER, W.J. y NELSON, M.A. (1996). "An IRS production function". National Tax Journal, 49(1), pp. 105-115.

IGLESIAS GÓMEZ, G. y SEIJAS DÍAZ, A. (2008). "Evaluación de la eficiencia productiva de los parques eólicos gallegos". Estudios de Economía Aplicada, Vol. 26(3), pp. 1-26.

JHA, R., et al. (1999). "Tax efficiency in selected Indian States". Empirical Economics, 24, pp. 641-654.

JIMÉNEZ, J. D. y BARRILAO, P.E. (2001). "Una aproximación a la eficiencia en la gestión de la Agencia Estatal de la Administración Tributaria". Papeles de Economía Española, 87, pp. 221-28. 
MAEKAWA, S. y ATODA, N. (2001). "Technical inefficiency in Japanese Tax Administration". $57 t^{\circ}$ Congreso del Instituto Internacional de Hacienda Pública, Linz, Austria.

MATTOS, E.; ROCHA, F. y ARVATE, P. (2011). "Flypaper effect revisited: evidence for tax collection efficiency in Brazilian municipalities". Estudios Económicos (São Paulo), 41(2), pp. 239-267.

MINISTERIO DE ECONOMÍA Y HACIENDA Y MINISTERIO DE HACIENDA Y ADMINISTRACIONES PÚBLICAS (2005 - 2013). "Informe sobre la cesión de tributos a las CCAA en cumplimiento de las medidas fiscales y administrativas del nuevo sistema de financiación de las CCAA de régimen común y ciudades con estatuto de autonomía. Ejercicios 2004 al 2012".

MOESEN, W. y PERSOON, A. (2002). "Measuring and explaining the productive efficiency of tax offices: a non-parametric best practice frontier approach". Tijdschrift voor Economie en Management, vol. XLVII, 3, pp. 399-416.

PÉREZ, C. (2012). Estadística Aplicada. España: Garceta Grupo Editorial.

RUY, S. y LEE, S. (2013). "An exploratory study of efficiency in tax jurisdictions". Advanced Science and Technology Letters, 34, pp. 46-49.

SAVIĆ, G.; et al. (2015). "Impact of the efficiency of the tax administration on tax evasión". Economic Research-Ekonomska Istrazivanja, 28(1), pp. 1138-1148.

SHEPARD, R.W. (1970). Theory of cost and production functions. Estados Unidos: Princeton University Press.

SIMAR L. y P.W. WILSON (1998). "Sensitivity analysis of efficiency scores: How to bootstrap in nonparametric frontier models". Management Science, vol. 44(1), pp. 4961.

THIRTLE C.; (2000). "Size does matter: technical and scale efficiency in Indian state tax jurisdictions". Review of Development Economics, 4(3), pp. 340-452.

TRILLO, D. (2002). La función de distancia: un análisis de la eficiencia en la Universidad. Tesis Doctoral, Departamento de Economía, Facultad de Ciencias Jurídicas y Sociales, Universidad Rey Juan Carlos.

TSAKAS, M. y KATHARAKI, M. (2010). "Assessing the efficiency and managing the performance of Greek Tax-Offices". Journal of Advances in Management Research, 7(1), pp. 58-75.

TSAKAS, M. Y KATHARAKI, M. (2014). "Impact of environmental factor son the efficiency of tax organizations". Serbian Journal of Management, 9(1), pp. 31-43.

\section{ABREVIATURAS}

ACAT: AGENCIA CÁNTABRA DE ADMINISTRACIÓN TRIBUTARIA

ACM: ANÁLISIS DE CORRESPONDENCIAS MÚLTIPLE

ACS: ANÁLISIS DE CORRESPONDENCIAS SIMPLE

AEAT: AGENCIA ESTATAL DE ADMINISTRACIÓN TRIBUTARIA

ATA: AGENCIA TRIBUTARIA AUTONÓMICA

ATIB: AGENCIA TRIBUTARIA DE LAS ISLAS BALEARES 
ATRIAN: AGENCIA TRIBUTARIA DE ANDALUCÍA

DEA: DATA ENVELOPMENT ANALYSIS, ANÁLISIS ENVOLVENTE DE DATOS

DEA-BCC: ANÁLISIS ENVOLVENTE DE DATOS -BANKER, CHARNES Y COOPER

DEA-CCR: ANÁLISIS ENVOLVENTE DE DATOS -CHARNES, COOPER Y RHODES

DMU'S: UNIDADES DE TOMA DE DECISIONES, DECISION MAKING UNITS

EM: ESCALAMIENTO MULTIDIMENSIONAL, MDS: MULTIDIMENSIONAL SCALING

FDH: FREE DISPONSAL HULL

IEDMT: IMPUESTO ESPECIAL SOBRE DETERMINADOS MEDIOS DE TRANSPORTE IP: IMPUESTO SOBRE EL PATRIMONIO

IPM: ÍNDICE DE PRODUCTIVIDAD DE MALMQUIST

ISD: IMPUESTO SOBRE SUCESIONES Y DONACIONES

ITPAJD: IMPUESTO SOBRE TRANSMISIONES PATRIMONIALES ONEROSAS Y ACTOS JURÍDICOS DOCUMENTADOS

CCAA: COMUNIDADES AUTÓNOMAS

IVA: IMPUESTO SOBRE EL VALOR AÑADIDO

IVMDH: IMPUESTO SOBRE LA VENTA MINORISTA DE DETERMINADOS

HIDROCARBUROS

LOFCA: LEY ORGÁNICA DE FINANCIACIÓN DE LAS COMUNIDADES AUTÓNOMAS

OCDE: ORGANIZACIÓN PARA LA COOPERACIÓN Y EL DESARROLLO

ECONÓMICO

RDBA: RENTA DISPONIBLE BRUTA AJUSTADA

SFA: STOCHASTIC FRONTIER ANALYSIS, ANÁLISIS DE FRONTERA ESTOCÁSTICA 


\section{Anexos}

Tabla 4

Descriptivos de las variables outputs pre-seleccionadas en la investigación DEA periodo 2004-2012

\begin{tabular}{|l|r|r|}
\hline & Media & $\begin{array}{r}\text { Desv. } \\
\text { típica }\end{array}$ \\
\hline 1. Gestión de liquidaciones complementarias del IP (unidades liquidaciones) & 144 & 319 \\
\hline 2. Gestión total de recursos del IP (unidades recursos) & 16 & 30 \\
\hline 3. Gestión total de expedientes del ISD (unidades expedientes) & 31.455 & 24.221 \\
\hline 4. Gestión total de recursos del ISD (unidades recursos) & 751 & 690 \\
\hline 5. Gestión total de autoliquidaciones del ITPAJD (unidades autoliquidaciones) & 416.964 & 381.882 \\
\hline 6. Gestión total de recursos del ITPAJD (unidades recursos) & 1.728 & 1.275 \\
\hline 7. Total de tasaciones periciales contradictorias (unidades de tasaciones) & 363 & 427 \\
\hline $\begin{array}{l}\text { 8. Gestión de las Tasas sobre el Juego de casinos y bingos (unidades } \\
\text { declaraciones) }\end{array}$ & 9 & 7 \\
\hline $\begin{array}{l}\text { 9. Gestión de las Tasas sobre el Juego de casinos y bingos (unidades cartones } \\
\text { vendidos) }\end{array}$ & 103.163 & 104.419 \\
\hline $\begin{array}{l}\text { 10. Gestión de las Tasas sobre el Juego máquinas o aparatos automáticos } \\
\text { (unidades autoliquidaciones) }\end{array}$ & 52.332 & 54.824 \\
\hline 11. Gestión de las Tasas sobre el Juego (unidades recursos) & 13 & 48 \\
\hline 12. Comprobación de valores declarados (unidades comprobaciones) & 74.909 & 89.581 \\
\hline 13. Actas de inspección instruidas (unidades actas) & 614 & 902 \\
\hline 14. Actas de inspección instruidas (miles de euros) & 18.805 & 24.164 \\
\hline 15. Recursos resueltos por actas de inspección (unidades recursos) & 27 & 36 \\
\hline 16. Expedientes sancionadores de inspección (miles de euros) & 3.078 & 3.965 \\
\hline 17. Expedientes sancionadores de inspección (unidades expedientes) & 367 & 537 \\
\hline 18. Derechos contraídos (miles de euros) & 1.275 .346 & 1.448 .994 \\
\hline 19. Recaudación aplicada líquida (miles de euros) & 9.718 & 11.259 \\
\hline 20. Providencias de apremio expedidas (unidades providencias) & 32.833 & 37.686 \\
\hline 21. Providencias de apremio expedidas (miles de euros) & & 1.329 .659 \\
\hline
\end{tabular}

Fuente: Elaboración propia con SPSS. 
Tabla 5

Descriptivos de las variables input - output utilizadas en el DEA periodo 2004-2012

\begin{tabular}{|c|c|c|c|c|c|c|c|c|c|c|c|c|}
\hline & \multicolumn{10}{|c|}{ Outputs } & \multicolumn{2}{|c|}{ Inputs } \\
\hline & \multicolumn{2}{|c|}{ 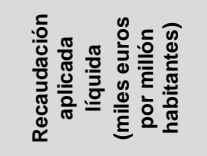 } & \multicolumn{2}{|c|}{ 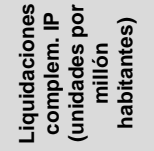 } & \multicolumn{2}{|c|}{ 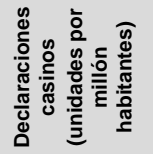 } & \multicolumn{2}{|c|}{ 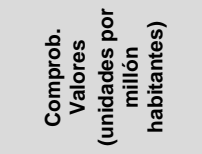 } & \multicolumn{2}{|c|}{ 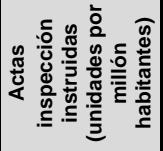 } & \multicolumn{2}{|c|}{ 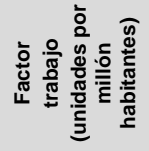 } \\
\hline & Media & $\begin{array}{l}\text { Desv. } \\
\text { típica }\end{array}$ & Media & $\begin{array}{l}\text { Desv. } \\
\text { típica }\end{array}$ & Media & $\begin{array}{l}\text { Desv. } \\
\text { típica }\end{array}$ & Media & $\begin{array}{l}\text { Desv. } \\
\text { típica }\end{array}$ & Media & $\begin{array}{l}\text { Desv. } \\
\text { típica }\end{array}$ & Media & $\begin{array}{l}\text { Desv. } \\
\text { típica }\end{array}$ \\
\hline Andalucía & 334.505 & 130.732 & 7 & 21 & 2 & 0 & 19.358 & 9.056 & 468 & 84 & 155 & 14 \\
\hline Aragón & 434.340 & 149.967 & 154 & 114 & 5 & 2 & 33.868 & 7.002 & 393 & 109 & 184 & 5 \\
\hline Asturias & 319.206 & 86.087 & 32 & 36 & 3 & 2 & 8.684 & 2.862 & 326 & 128 & 130 & 15 \\
\hline Baleares & 548.097 & 219.870 & 317 & 299 & 11 & 1 & 10.549 & 4.497 & 311 & 127 & 142 & 23 \\
\hline Canarias & 272.650 & 101.004 & 19 & 17 & 13 & 1 & 28.426 & 10.447 & 110 & 62 & 176 & 15 \\
\hline Cantabria & 437.095 & 147.769 & 14 & 26 & 5 & 3 & 32.298 & 8.672 & 282 & 119 & 159 & 12 \\
\hline Cataluña & 505.238 & 219.597 & 22 & 18 & 2 & 0 & 5.011 & 1.380 & 47 & 14 & 99 & 4 \\
\hline Castilla y León & 309.453 & 113.463 & 12 & 30 & 5 & 0 & 137.670 & 20.998 & 227 & 86 & 195 & 10 \\
\hline Castilla Ia Mancha & 313.812 & 115.622 & 19 & 21 & 0 & 0 & 48.243 & 11.133 & 83 & 35 & 162 & 9 \\
\hline Extremadura & 205.594 & 52.969 & 8 & 11 & 3 & 2 & 23.999 & 9.451 & 195 & 126 & 156 & 12 \\
\hline Galicia & 267.923 & 86.419 & 35 & 27 & 3 & 0 & 53.001 & 6.549 & 276 & 55 & 172 & 4 \\
\hline La Rioja & 424.294 & 156.942 & 693 & 370 & 10 & 3 & 79.178 & 24.191 & 469 & 137 & 182 & 13 \\
\hline Madrid & 534.523 & 244.993 & 133 & 143 & 1 & 0 & 11.194 & 2.019 & 117 & 19 & 92 & 3 \\
\hline Murcia & 380.419 & 185.204 & 11 & 18 & 6 & 0 & 16.177 & 2.529 & 383 & 92 & 184 & 8 \\
\hline Valencia & 413.985 & 201.407 & 18 & 21 & 4 & 1 & 10.192 & 2.123 & 68 & 9 & 128 & 6 \\
\hline Media & 380.076 & 147.470 & 100 & 78 & 5 & 1 & 34.523 & 8.194 & 250 & 80 & 154 & 10 \\
\hline
\end{tabular}

Los estadísticos descriptivos se ofrecen en términos relativos -por millón de habitantes-, para ser comparables entre CCAA. No obstante, las variables empleadas en el DEA se expresan en términos absolutos por tratarse de un análisis de optimización matemática de ratios.

Fuente: Elaboración propia con SPSS. 